\title{
Immobilization tools and materials selection in dentoalveolar fracture
}

\author{
Endang Samsudin \\ Department of Oral and Maxillofacial Surgery Faculty of Dentistry Universitas Padjadjaran \\ Hasan Sadikin Hospital-Bandung, West Java, Indonesia
}

\begin{abstract}
Dentoalveolar fracture treatment can be performed in a simple way without surgery by reposition the fractured fragment and establish immobilization. One of the factors affecting the success of dentoalveolar fracture treatment is immobilization material and technique selection. The purpose of this study is to provide illustration about various materials used to perform immobilization in dentoalveolar area and the objective is to obtain optimal immobilization.
\end{abstract}

Key words: Immobilization tools, dentoalveolar fracture, fixation, reduction

\section{INTRODUCTION}

Dentoalveolar fracture is a fracture on teeth and supporting alveolar bone both in maxilla and mandible. The fracture is usually related to the type of teeth and jaw bone. Alveolar bone fracture often happens in damaged teeth, such as crown and root fractures, and the presence of soft tissue lesion. ${ }^{1,2}$

Dentoalveolar fracture is commonly found in child anterior teeth, especially in maxillar denture. This lesion is often caused by other teeth or facial traumas. Dentoalveolar fracture can be caused by direct dental trauma or indirect trauma of the chin. ${ }^{1}$ Children with protrusive anterior teeth have a predisposition of dentoalveolar trauma. ${ }^{3}$ Dentoalveolar fracture frequency in mandible is $1-5 \%$, while dentoalveolar fracture incidence in school-age child is $5 \% .^{1}$ Dentoalveolar fracture etiology generally consists of trauma caused by fighting, traffic accident, accident during exercise or playing and fall. ${ }^{4}$ Dentoalveolar fracture in children often serves as an effect of trauma occurred in accidents in school and house. ${ }^{5}$

Dentoalveolar trauma is an unpleasant experience for children and adult. Trauma of the face resulted in dentoalveolar fracture may causes dental displacement or dental loss that may inflict the function and the esthetics during facial development. Failure in dentoalveolar fracture treatment is usually found when the treatment is too late and inappropriate immobilization technique and tools/material selection are utilized. Correct and immediate case management should improve the success level of this treatment.

Dentoalveolar fracture treatment should reduce and return the fracture segment to the right position and fixed it in place until the bone starts to recover. Reducing fracture segment is performed by giving pressure on the bone and teeth segment digitally. Correct immobilization tools and technique selection will accelerate the process and increase the success level. ${ }^{1,2}$ 


\section{DENTOALVEOLAR FRACTURE}

\section{Fracture classification}

Many systems have been developed to classify types of dentoalveolar trauma. WHO system modified by Andereasen has classified dentoalveolar trauma which includes dental, supporting structure, oral gingival and mucosa traumas according to the anatomy, therapist consideration and prognosis. ${ }^{6}$ Sanders et al. modified WHO dentoalveolar trauma classification based on the cause and time, related dental structure, and type of displacement as well as root and crown fracture. ${ }^{2}$ Several fracture classification examples are partial enamel fracture, horizontal and vertical crown fracture, crown-root fracture, horizontal root fracture, dental mobility, dental displacement, avulsion, and alveolar process fracture. Clark ${ }^{4}$ specifically classified alveolar fracture into e: alveolar fracture Class I in nondental area, alveolar fracture Class II in dental area with small displacement, alveolar fracture Class III in dental area with medium-heavy displacement, alveolar fracture Class IV with several fracture lines related to others fractures in dental supporting jaw bone.

\section{Clinical symptoms and examination}

To uphold correct-dentoalveolar fracture diagnosis, it is necessary that accurate anamnesis and clinical examination are perform. The initial stage to perform trauma history anamnesis comprehensively consists of patient personal data, when, where, how the trauma happens, what treatment performed initially, is there any tooth or tooth fracture at the location, patient's general condition, is there any systemic disease history, and is there any mastication disorder. ${ }^{2}$

Next, the clinical examination is performed carefully and accurately. Treatment of dentoalveolar trauma patient does not only require examination on the related structure but also the vital signs such as pulse, blood pressure and respiration and these can be performed simultaneously during anamnesis. During the clinical examination, there are several things that should be examined routinely including extra and intra-oral soft tissue injury, jaw or alveolar process fracture, dentalcrown examination, dental displacement, dental mobility, percussion or periodontal disorder, and dental vitality. ${ }^{1,2}$

Dentoalveolar fracture clinical signs include dental displacement and loosening in one segment, percussion pain, laceration in gingival and lips vermilion, echimosis in vestibulum, and inflammation or chin injury. ${ }^{7}$

\section{Radiograph analysis}

Radiograph analysis is very useful in diagnosing dentoalveolar fracture. Several $\mathrm{x}$-ray techniques that are already familiar in radiograph analysis of dentoalveolar trauma cases include periapical imaging, oclusal imaging, panoramic imaging, and, in some cases, posteroanterio or lateral imaging as required.

Radiographimage should provide information about root fracture condition, intrusion or extrusion level, disorder presented in periapical, root development, pulp chamber size, root canal, jaw fracture involvement, root fragment and foreign substances in soft tissue. ${ }^{2,7}$

\section{Treatment}

The purpose of dentoalveolar trauma treatment is to return the normal function and shape of the mastication organ. Dentoalveolar trauma treatment includes 2 stages, i.e. immediate treatment following trauma (emergency treatment) and treatment for teeth or alveolar bone with trauma. Emergency treatment is very important and should be performed as soon as possible because it relates to prognosis. This treatment includes cleaning, suturing, pain removal and infection prevention.

The definitive treatment is the repositioning the alveolar or teeth with trauma and maintaining it in place until fixation is achieved. Reposition technique for teeth or alveolar fragment with fracture/displacement can be performed by fingers or instruments. Period of immobilization depends on the damage level of the dental and supporting tissue, dental apex condition, immobilization material and technique. Usually, the period of immobilization in dentoalveolar fracture ranges between 7 days and 6 weeks. ${ }^{2,7}$

\section{Immobilization materials and methods}

There are several immobilization materials and methods of dentoalveolar fracture. Good material and method for fixation and immobilization 
should meet these following criteria: ${ }^{1}$ Material and tools should be easily installed without previous laboratory procedure; Passive in it location without giving pressure on teeth; Do not contact or irritate the gingiva; There is no hook during normal occlusion; Easy to be cleaned and used in good oral hygiene; Do not cause trauma to teeth and gingiva during use; Allow endodontic treatment; Easy to remove/release; and Have good esthetics.

\section{Foil splint}

In emergency, it is very possible to use a splint made of film or cigarette foil. This material can be easily applied and warp the sub-luxated tooth along with adjacent tooth. In order to obtain more rigid result, two layers of this splint may be cemented on the tooth.

\section{Composite resin splint}

In order to immobilize dentoalveolar fracture, the composite resin is applied and bound to the enamel surface of the loose tooth to several sound teeth as the abutments.

\section{Composite with Nylon or floss}

Nylon or dental floss are adapted to each loose teeth and sound teeth, and then fixated by acid-etch Composite. This material fixation can be performed on occlusal or tooth labial surface. The composite surface is softened and polished to reduce food debris retention.

\section{Composite with wire}

Stainless steel wire with a diameter of 0.3 $\mathrm{mm}$ is bound to several loose teeth to the sound teeth as the abutments, and then it is fixated on each teeth using light-cure composite.

\section{Acrylic splint}

Acrylic can be used as dentoalveolar fracture immobilization tool. This material can be easily applied and is beneficial in emergency of dentoalveolar fracture.

\section{Arch bars}

This immobilization tool is made of metal that can be adapted to the arch and bound to teeth using stainless steel wire with a diameter of $0.3 \mathrm{~mm}$ or $0.4 \mathrm{~mm}$. This tool is benefitcial for maintaining the alveolar fracture and dental subluxation and loaded teeth. This tool can retain alveolar fragment that tends to move occlusally and may be used for labial support of sub-luxated tooth.

\section{Various splinting techniques using wire}

Usually, wire with a diameter of $0.3 \mathrm{~mm}$ or $0.4 \mathrm{~mm}$ and $0.5 \mathrm{~mm}$ is used as amplifier. The binding technique used can be in the form of number 8 model, using loop, eyelet or ivy.

\section{Orthodontic tools}

Tools for orthodontic treatment such as orthodontic Band, bracket and arch wire can be used for sub-luxated tooth immobilization to the adjacent tooth.

\section{DISCUSSION}

Dentoalveolar fracture often accompanies trauma of face and head. The most protruding part of the face is teeth and jaw bone, so the trauma in this area usually results in dentoalveolar fracture. The main causal factors of dentoalveolar fracture include traffic and exercise accidents, and fighting. ${ }^{1,4}$

If the treatment is not immediately provided, then it is possible for complication such as bleeding, infection, tissue necrosis, and malocclusion, to occure and it will eventually disrupt the esthetics. This delay is usually caused by the fact that patient does not go to the medical centre right away or insufficient dental skill of the dentist in treating teeth or jaw trauma.

Dentoalveolar fracture clinical signs include loosening or displacement of tooth or teeth in a segment, malocclusion, percussion pain, laceration in gingival and lips vermilion, echimosis in vestibule, and inflammation or chin injury. ${ }^{7}$ In confirming dentoalveolar fracture, accurate anamnesis and clinical examinations, as well as radiograph analysis are needed. X-ray images taken in dentoalveolar trauma includes periapical image, occlusal image, and panoramic image. Fracture line that separates the fragments in radiograph image can be seen as radiolucent line on the hard tissues (teeth and bone).

In reality, dentoalveolar fracture treatment is quite simple, which is reducing and returning 

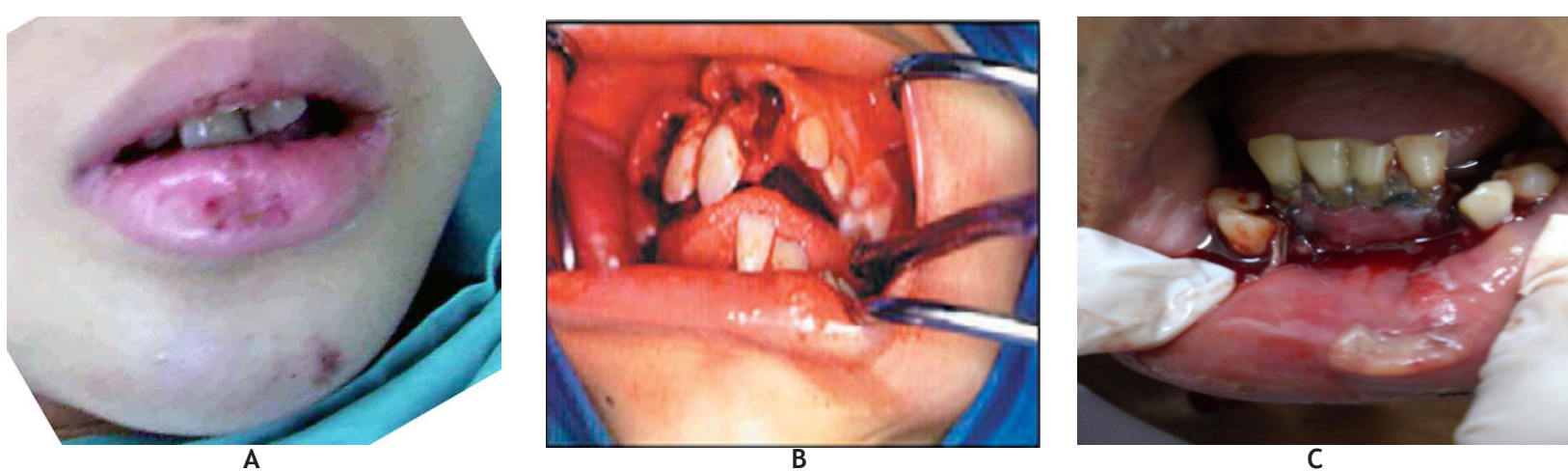

Figure 1. Dentoalveolar fracture clinical signs: gingival injury, tooth malposition, malocclusion, tooth avulsion, mobility.
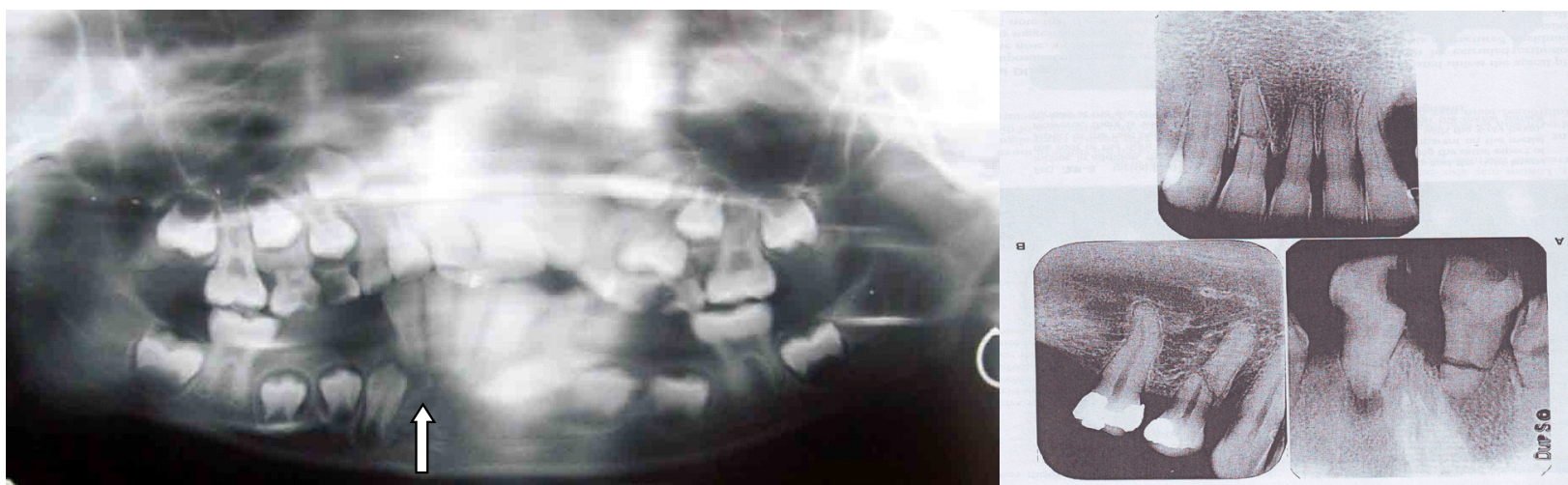

Figure 2. Radiograph analysis is very useful in diagnosing dentoalveolar fracture.

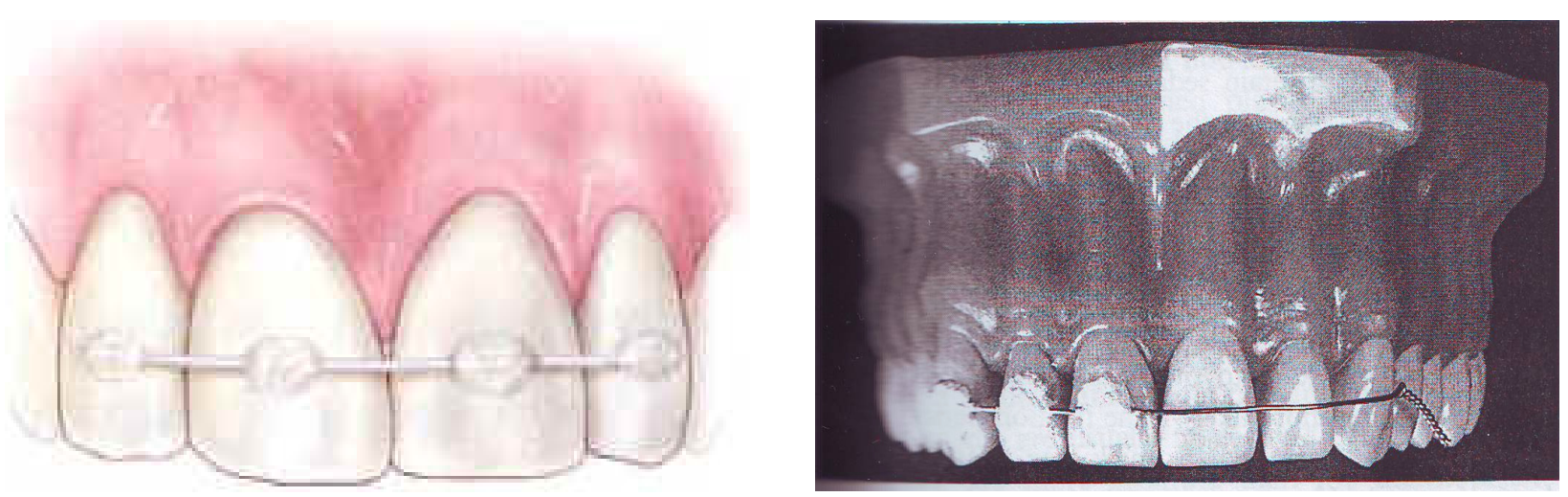

Figure 3. Fixation using composite and wire.
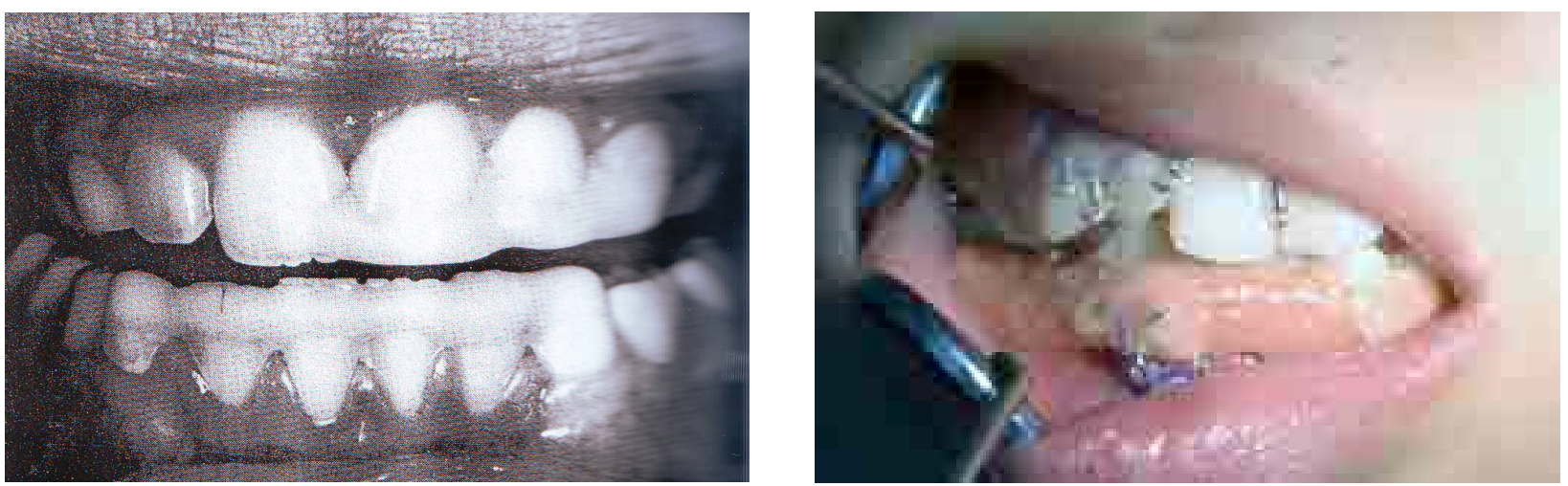

Figure 4. Acrylic splint utilization. 

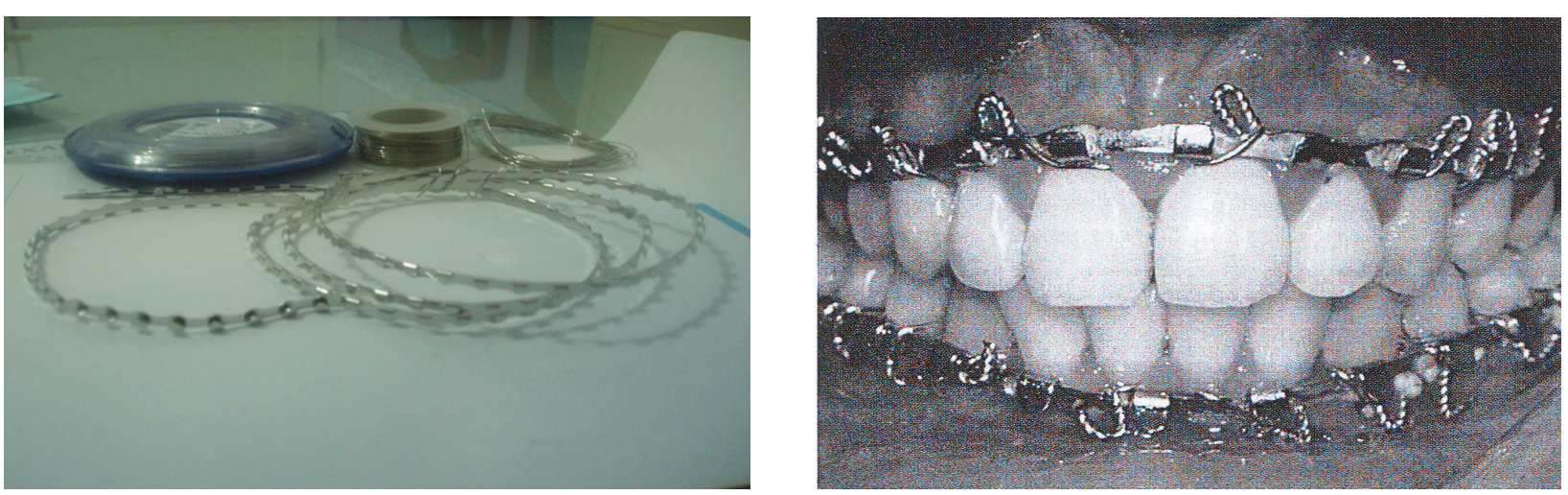

Figure 5. Immobilization using arch bars.
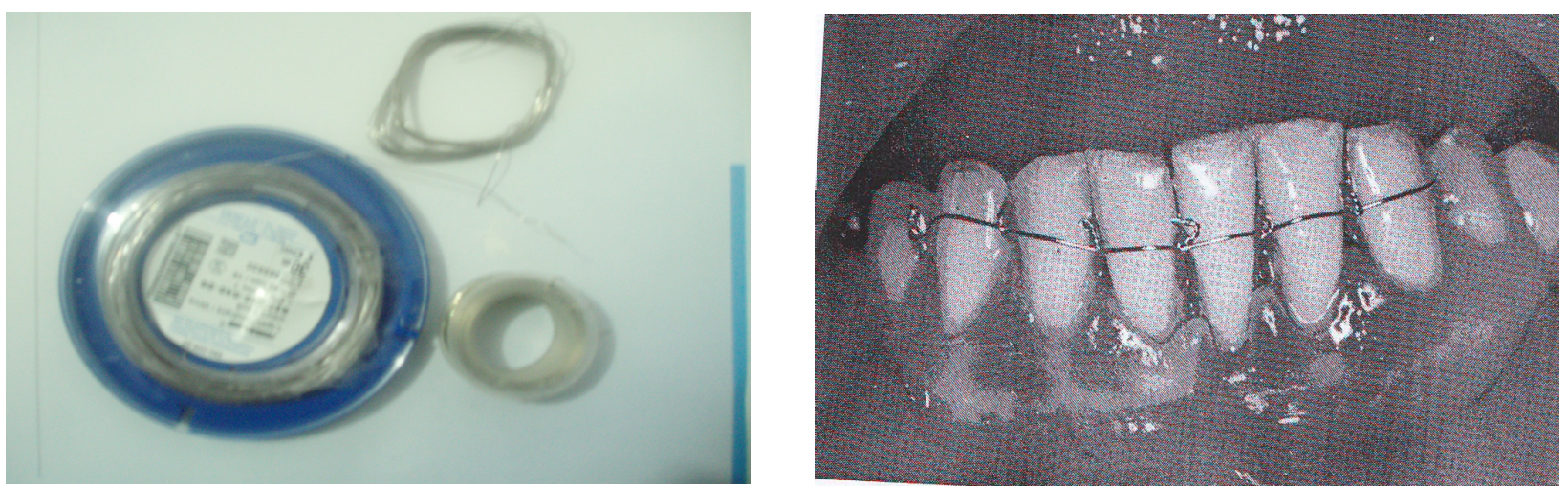

Figure 6. Splinting technique immobilization using wire.

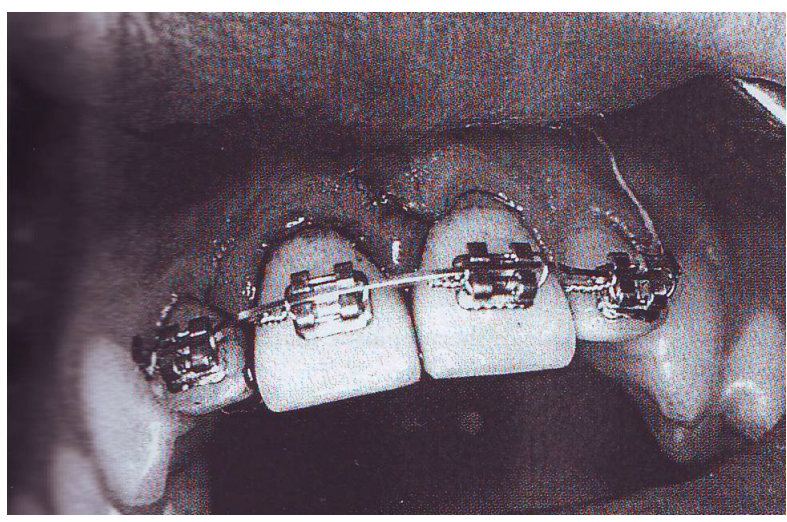

Figure 7. Immobilization using orthodontic tools.

the fracture segment to the correct position and fix it in place until the bone recovers. Reducing the fracture segment can be performed by giving pressure digitally on teeth or bone segment using hand. While for fixation and immobilization, there are various tools that can be utilized. ${ }^{1,2}$

The immobilization tools and methods selection depend on the tissue damage level, morphology and the dental development. In emergency situation where dentoalveolar affects several teeth, foil and acrylic splints can be used temporarily for immobilization while the patient is referred to a medical centre with more sufficient facilities. This material can be applied on permanent and primary teeth. Acrylic splint is not quite strong against occlusal force, so it usually loosens and moves apically.

Composite resin material can be used for dentoalveolar fracture immobilization with mild tissue damage level. This material is used on several teeth with moderate mobility. This material also has good esthetic because the color can be adjusted to the tooth color. This composite resin can be combined with Nylon, dental floss or wire. This method is very simple and efficient because it does not need anesthesia, good hygiene, and can be used in definitive treatment. The material is a semi-rigid splint that can stabilize the trauma tooth and give opportunity for physiologic movement of the teeth that will eventually minimize root anxylosis and resorption. ${ }^{1}$

Arch bar is a very good immobilization tool for dentoalveolar fracture case with extended damage. This material is quite strong to resist mastication-load. However, it has several 
weaknesses such as poor esthetic and it often causes residual food retention and pressure to gingiva. Therefore, this material is unfavorable from the hygienic point of view. This immobilization tool installation also needs special skill. Splinting with stainless steel is generally easy to use and quite strong for dentoalveolar fracture immobilization in several teeth.

\section{CONCLUSION}

The success of dentoalveolar fracture treatment will be achieved if it is performed immediately and if accurate immobilization methods and tools are used. Immobilization tool and method selection depends on tissue damage level, morphology, and dental development. Dentists should understand the dentoalveolar fracture immobilization simple method for emergency.

\section{REFERENCES}

1. Fonseca RJ, Walker RV, Betts NJ, Barber HD.
Oral and maxillofacial trauma. Vol.1. $3^{\text {rd }}$ ed. Philadelphia: W.B. Saunders Co.; 2005.

2. Peterson LJ. Contemporary oral and maxillofacial surgery. $3^{\text {rd }}$ ed. St. Louis. Livingstone; 2003.

3. Kaban LB. Pediatric oral and maxillofacial surgery. Philadelphia: W.B. Saunders Co.; 1990.

4. Clark WD, Park G. Fractures mandibular alveolar. 2002. Available from: http://www. emedicine.Com/ent/topic164.htm.

5. Banks K, Merlino P. Minor oral injury in children. 1998 Available from: http://www. mssm.edu/ms.journal/65/04.Banks.pdf.

6. William J, Rowe LI. Williams' maxillofacial injuries. $2^{\text {nd }}$ ed. London: Churchill Livingstone; 1994.

7. White SC, Pharoah MJ. Oral radiology, principles and interpretation. $5^{\text {th }}$ ed. St. Louis: Mosby; 2004.

8. Camp J, Stewart C. Dental trauma. Available from: File://A:IEMR.Textbook-Dental Trauma. htm.

9. Pedersen G. Buku ajar bedah mulut. Jakarta: EGC; 1996. 